\title{
Exploration of Rural Landscape's Significance in Landscape Architecture Planning and Design
}

\author{
Zhoumin Yang \\ Art design college, Xi'an Peihua University, Xi'an Shanxi, 710125, China
}

Keyword: Landscape Architecture, Planning and Design, Rural Landscape, Significance.

\begin{abstract}
During recent years, Chinese landscape architecture planning and design has gradually shifted the emphasis from the urban areas to rural areas. Construction of rural landscape has begun to be highly valued by the designing field, further to increasingly develop into the new characteristics and thought of the landscape architecture design. To properly integrate designing elements of rural landscape in the planning and design of landscape architecture, it can fatherly enrich the contents of landscape architecture planning, reinforce the diversity of designing and promote the designing level to be consistently improved. This paper analyzes the principles and significance of integrating rural landscape into the current landscape architecture planning and design, and also puts forward corresponding suggestions, with the hope of offering good supports for the development of landscape architecture planning and design.
\end{abstract}

\section{Introduction}

With the construction and development of Chinese socialist market economy, Chinese society has consistently made progress. People's living standard has also been obviously improved. Under the stimulation of rich material life, people have started to pursue more qualified spiritual life, whose consciousness of environment and appreciation of aesthetics has also been properly improved, further to have certain impacts on the planning and design of urban landscape architecture. In the New Era, affected by the urban residents’ psychology of longing for peaceful rural life, to properly integrate rural landscape into the planning and design of landscape architecture has also gradually developed into the key point of planning and design. In addition, good integration can also promote the landscape architecture planning and design to obtain certain aesthetic charm, which may even create certain opportunity of sustainable development. Therefore, in the New Era, combined with the principles of landscape architecture planning and design, exploration of the significance and methods of integrating rural landscape into the planning and design of landscape architecture has certain realistic value, which is worthy of deeper exploration and researches.

\section{Principles to be Followed while Integrating Rural Landscape into Landscape Architecture Planning and Design}

To properly integrate rural landscape into landscape architecture planning and design, three principles should be stick to:

First of all, it must insist on the principle of openness during the development process. Principle of openness is the fundamental one that all landscape architecture planning and design should follow. Only by sticking to the opening principle, can it guarantee that products can conform to the aesthetic appreciation of modern people and rules of the nature, can it reflect the changes of the present age and the major development designing trends in future. Therefore, to integrate the rural landscape into landscape architecture planning and design, it should strengthen the emphasis on the opening to the public. Only by opening to the public and the whole society, and also exploring diversified artistic manifestation forms, can it increase the depth and wideness of the artistic design, further to actually integrate rural landscape into landscape architecture planning and design.

Secondly, it should insist on the principle of coordination. Generally speaking, rural landscape actually contains a lot of contents, such as the performance of geomorphology, continuation of 
culture, reflections on history and reinforcement of ecological protection. Consequently, to integrate rural landscape into landscape architecture planning and design, it must pay attention to reasonably coordinate all contents of the rural landscape, so as to guarantee all functions of rural landscape to be played and realize the coordinated development among all elements. Besides, it should also attach importance to the concept of general situation during the integrating process, reinforce the organic coordination between rural landscape element and other surrounding element, so as to actually improve the artistry of landscape architecture and promote it to be recognized by more people ${ }^{[1]}$.

Lastly, it should also stick to the principle of integrating planning with protection. To properly integrate rural landscape into landscape architecture planning and design, it is just one effective utilization and protection for natural resources and resources characteristic by the rural areas. To analyze that in a certain sense, all resources involved in the integrating process are just those resources damaged by the urban industrialization construction. Thus rational integration of rural landscape should also pay attention to integrate planning with protection. During the designing process, it should protect the historical and cultural characteristics of all regions, inherit the historical and cultural traditions, and also create conditions for carrying forward and developing traditional national culture.

\section{Significance of Integrating Rural Landscape into Landscape Architecture Planning and Design}

\section{Representing Local Customs and Practices of the Society}

During recent years, with development of Chinese economic society and accelerated urbanization construction, urban reinforced concrete architectures have gradually become the mainstream, which have certain impacts on people's life and make people more long for the green rural life. Under the influence of such a concept, rural landscape has been gradually introduced into the landscape architecture planning and design. Local customs and practices, historical culture and characteristic natural landscapes of the rural areas are represented in the city by another way. In the meanwhile of enriching the urban landscape, it can also realize the inheritance of various urban cultures, show the protection of rural historical customs and reflect the local customs and practices of the society. In this way, people living in cities can feel the non-urban sense of space and nature by appreciating the rural landscapes, so as to return to nature and experience the beautiful life.

\section{Organically Integrating Economic Development with Environmental Beautification}

Currently, in the planning and design of landscape architecture, various designing elements have promoted the landscape architecture to show diversified development characteristics to a certain extent. Meanwhile, in order to guarantee the character of era and artistry of landscape architecture planning and design, the construction budget has also consistently increased. Due to insufficient economical efficiency of landscape planning, it has produced bad influence on the continuous development of landscape architecture. To integrate rural landscape into landscape architecture planning and design can adequately improve such a situation. Because rural landscapes more conform to the aesthetic requirements of the audiences, more tourists can be attracted by the gardens. Economic benefits of landscape architecture can be gradually highlighted. It can be seen that, to integrate rural landscape into landscape architecture planning and design can organically integrate the economical efficiency with the beautification. In the meanwhile of promoting the economic development of gardens, it can also satisfy the landscape requirements of the audiences as well, so as to fatherly improve the quality of landscape architecture planning and design.

\section{Properly Increasing the Audiences’ Agricultural Experience by Applying Rural Landscapes}

To properly integrate rural landscape into landscape architecture planning and design can let the audiences adequately increase their own agricultural experiences during the process of admiration and feeling, help people living in cities who barely contact with agriculture to form more profound understandings about the agricultural production and enrich the spiritual life ${ }^{[2]}$. In this way, the 
audiences can actually feel the development histories of agriculture and regions conveyed by the landscape architectures, and also obtain certain agricultural experiences, further to promote Chinese construction of agricultural society to be understood by more people and lay a foundation of manpower for the agricultural development.

\section{Measures of Reasonably Integrating Rural Landscapes into Landscape Architecture Planning and Design}

There are usually two ways to integrate rural landscape into landscape architecture planning and design. The first one is simulation. People can plan and design gardens with rural landscape characteristics in the cities dominated by reinforced concrete architectures, so as to create certain leisure and entertainment environment for urban residents. The second is borrowed scenery. During the process of urban planning, people can plan those rural remains with certain rural landscape characteristics as the gardens, or extend gardens to the rural areas properly, further to integrate the landscape planning with rural landscapes, and then reinforce the rural characteristics of landscape architecture. In addition, with the help of good design and planning, the rural landscapes can be promoted to be coordinated with the overall landscape planning, so as to highlight the humanity, artistry, ecology and nature of rural landscapes.

\section{While Integrating the Rural Landscapes, People should Pay Attention to the Extension of Rural Historical and Cultural Heritage.}

During the long development process, historical and cultural heritages accumulated in rural areas show the historical culture with rural characteristics to a certain extent. Therefore, during the process of landscape planning, in order to realize the perfect integration with rural landscape, people should strengthen the protection and inheritance of rural historical culture, really realize the cohesion of rural flavor, so as to promote the entire rural geographical landscapes to be represented in the planning and design of landscape architecture. During the urban planning process, if designers integrate the rural landscapes by borrowed scenery, they can take advantage of the regional rural landscapes by designing the landscape architecture, further to present the characteristics of the rural landscape by regional landscapes, and then increase the rural atmosphere of landscape planning and design ${ }^{[3]}$. For instance, with the help of borrowed scenery, designers can properly extend the landscape architectures to the border of rural areas during the planning process, and also reserve certain access to the rural areas. People living in cities can directly appreciate a large area of forests, architectures with rural characteristics and agricultural atmosphere while visiting the landscape gardens. At this moment, in order to protect the rural ecology, designers cannot integrate a large area of landscape architectures with the rural areas, but open one corresponding land for constructing Agritainment in the garden, combined with the rural characteristics, thus tourists can experience the rural life here. In this way, it cannot only save costs, but also let people feel the most authentic agricultural life. In the meanwhile of deepening people's understandings about the local customs and practices of rural areas, and also the historical culture, promoting people to subjectively inheriting the historical and cultural heritage of rural areas, they can also protect the agricultural ecological environment. Moreover, economic benefits of the landscape architectures can be highlighted to a certain extent. It can be seen that, there is certain realistic significance to properly integrate rural landscapes into the landscape architectures.

\section{While Integrating the Rural Landscapes, People should Pay Attention to Increase the Agricultural Experience and Present the Idyllic Scenery}

At the present stage, because the urban life has been gradually separated from the rural life, people born in cities usually do not or barely contact with the agricultural work. Some children of the modern cities cannot understand the significance of farming, or form deep understandings about the normal process of agricultural production (sowing seed in Spring, growing in Summer, harvesting in Autumn and storing in Winter) ${ }^{[4]}$. Therefore, in order to implement universal agricultural education to people 
living in modern cities, designers should properly integrate rural landscapes into the planning and design of landscape architecture. Moreover, designing of landscape should emphasize on increasing agricultural experiences, so as to let people feel aspects of rural life and work from the urban gardens, increase the agricultural experiences of the audiences and promote the comprehensive quality of city dwellers to be fatherly improved. On that basis, while integrating the rural landscapes into the planning and design of landscape architecture, designers can open some agricultural experience pavilion and lands. In the meanwhile of appreciating the rural landscapes and culture with rural characteristics, people can also form deeper understandings about the agricultural life and culture by certain rural life experience, farming experience and harvest experience, so as to fatherly improve the comprehensive qualities of city dwellers and make certain contributions to the construction of harmonious society in China. For instance, during the process of integrating rural landscapes into planning and design of landscape architecture, designers should combine with the overall style of the landscape architecture and requirements of the special agricultural experiences, properly construct Peach Blossom Village and Almond Flower Village in landscape architectures, integrate the construction of scenery with the rural-characteristic life, and also set some nursing and experiencing zones of peach and almond, experiencing zone for picking peaches and almonds, so as to create proper experiencing life for city dwellers and create better conditions for giving the play to the agricultural experiencing value in landscape architectures.

\section{Landscape Architecture and Rural Landscape should be Uniformly Planned and Designed, further to Increase the Ecological and Economic Benefits of Landscapes.}

At present, with the updated planning idea of landscape architecture and reinforced planning quality, the designing of landscape architecture has shown more obvious openness. Some landscape architectures have even shown certain characteristics of half-openness. Therefore, while integrating the rural landscape into planning and design of landscape architecture, designers should make unified planning and design. Combined with the openness of landscape architecture, they can comprehensively promote the organic unification of ecological benefit and economic benefit. In the meanwhile of guaranteeing the ecology of landscape architecture, ecological propaganda effect of landscape architecture can be played to a certain extent. Moreover, with the help of rural landscape, it will increase the landscape architecture's appeal to the social public, increase the economic benefits of gardens and lay a foundation for the development of urban landscape architecture ${ }^{[5]}$. For instance, the 5A scenic spot of Henan Yuntai has integrated the rural landscape into the planning and design of landscape architecture, further to really realize the planning style of "household in scenic spot". While planning the landscape, designers do not remove all villages from the scenic spot, but reserve some villages. After the construction of scenic spot is completed, some villagers are still engaged in traditional labors, and the others carry out some operational activities related to the development of scenic spot. In this way, it cannot guarantee the continuation of village landscape, but also increase the living standard of villagers and attract tourists, further to promote the Yuntai spot to obtain better development. Meanwhile, in order to increase the economic efficiency of scenic spot, designers also pay attention to construct a large comprehensive entertainment place integrated of eating, lodging and traveling. Tourists can eat and get accommodation in rural home inns in the scenic spot. Moreover, they can also have proper entertainment with farmers and experience the rural flavor, so as to actually realize the organic integration of villages and scenic spots, promote the villages to play its biggest role in the planning and design of landscape architecture, and then create conditions for the better development of landscape architecture.

\section{Conclusion}

Above all, to integrate rural landscape into planning and design of landscape architecture can realize the inheritance and development of rural culture, represent the local customs and practices of villages, enrich the spiritual life of city dwellers and also let them obtain certain agricultural experiences. Therefore, landscape designers should attach more importance to the integrating work of rural 
landscape, promote landscape architecture to be recognized by more social groups by properly integrating rural landscapes into planning and design of landscape architecture, and then fully promote the planning and design of landscape architecture to obtain better development in the New Era.

\section{References}

[1] Wang Youlin. Significance of Rural Landscape in Planning and Design of Landscape Architecture, China Homes, 2012(19):3-4.

[2] Chen Ya. Studies on the Significance of Rural Landscape in Planning and Design of Landscape Architecture, New Material New Decoration, 2014(11):330-330.

[3] Hu Miao. Analysis on the Significance of Rural Landscape in Planning and Design of Landscape Architecture, Beijing Agriculture,2015(23):75-76.

[4] Chen Leishen. Significance of Rural Landscape in Planning and Design of Landscape Architecture, Xiandai Horticulture, 2015(8):101-102.

[5] Fan Jiaqi. Explorations to Integrate Rural Landscape into Planning and Design of Landscape Architecture, Art Science and Technology, 2015(10):234-234. 